\title{
LOCALIZACIÓN EN REDES Y SIMULACIÓN DE REDES DE PETRI APLICADAS A LA ATENCIÓN DE POBLACIONES AFECTADAS POR DESASTRES NATURALES
}

\author{
Esther Berger Vidal ${ }^{1}$, Inés Gambini López ${ }^{2}$ \& Jaime Alcalde Chigne ${ }^{3}$
}

Resumen: El objetivo de la investigación es minimizar el tiempo de atención a la población afectada por un desastre natural. Se plantea que la población afectada acuda a lugares seguros de refugio y atención de necesidades básicas, establecidos por la sede de defensa civil, cuyo conocimiento debe ser de permanente difusión entre la población. El estudio consta de dos fases, en la primera fase se determina la localización de los lugares de atención haciendo uso de redes de nodos tales que los nodos de una red representan las áreas donde reside la población. Se utiliza el modelo de las p-medianas y el criterio del inicio del tiempo de atención a los pobladores que acuden al centro de atención, asociado a la menor distancia para llegar al lugar de atención. Luego de haberse ubicado los lugares de atención, se enfoca el problema de la atención eficiente y oportuna a los pobladores que la requieren, para lo cual se construye una red de Petri para cada centro de atención. En esta red se representa el proceso integral de atención y todas sus alternativas: registro de personas, atención médica, refugio, entre otros servicios. La red de Petri resultante se simula para determinar las longitudes máxima y promedio de colas, tiempo promedio de espera, tiempo promedio en el proceso por persona y se realizan cambios en los parámetros para determinar su valor más adecuado para brindar la mejor atención posible. También se realizan cambios en los parámetros tales como tasa promedio de atención o incremento de puestos de atenciọ́n en cada lugar de atención y se determina el número óptimo de estos lugares para minimizar el tiempo de atención a la población afectada que acude a un refugio determinado. Este es un estudio en proceso cuyos resultados finales dependen de la disponibilidad de datos e información relacionada con el caso analizado relacionado con un sismo de alta intensidad.

Palabras clave: Localización, Redes de Petri, Simulación.

\section{LOCALIZATION IN NETS AND PETRI NET SIMULATIONS APPLICATED TO THE ATTENTION OF AFFECTED PEOPLE BY THE NATURAL DISASTERS}

\begin{abstract}
The research objective is to minimize the time to care for the population affected by natural disaster. It is suggested that the affected population go to safe places of refuge and care of basic needs, established by the civil defense headquarters, whose knowledge must be disseminated throughout the population permanently. The study consists of two fases, in the first stage determines the location of sites of care by using networks of nodes such that nodes of a network represent areas where the
\end{abstract}

\footnotetext{
${ }^{1}$ UNMSM, Facultad de Ciencias Matemáticas, e-mail: ebergerv@unmsm.edu.pe

${ }^{2}$ UNMSM, Facultad de Ciencias Matemáticas, e-mail: igambinil@unmsm.edu.pe

${ }^{3}$ UNMSM, Facultad de Ciencias Matemáticas, e-mail: jaimealcaldechigne@gmail.com
} 
population resides. It uses the model of the p-medium and the test of time home care to the residents who come to the center of attention, associated with the shortest distance to reach the point of care. After having located the sites of care, focuses the problem of efficient and timely care to the people that need it, for which we construct a Petri net for each focus. This network represents the whole process of attention and all its alternatives: registration of persons, medical care, shelter, among other services. The resulting Petri net is simulated to determine the maximum and average lengths of queues, average wait time, average time spent per person in the process and make changes in the parameters to determine their most appropriate value to provide the best care possible. Also make changes in parameters such as average rate of increase in attention or care posts at each point of care and determining the optimal number of these sites to minimize the time to care for the affected population who go to a specific shelter. This is a study underway with final results depend on the availability of data and information relating to the present case related to an earthquake of high intensity. Key words: Location, Petri nets, Simulation.

\section{Introducción}

Se trata de atender en el menor tiempo posible a la población afectada por un desastre natural. En particular en esta investigación hemos sido motivados por el último sismo de alto grado ocurrido en nuestro país en el año 2007, en el cual observamos que la atención a la población afectada no se realizó con la celeridad requerida, tal vez porque no se estimaba que pudiera producirse un sismo de tan alta intensidad e impacto. El sistema de defensa civil que prepara permanentemente a la población para este tipo de eventos y está apto para contrarrestar sus efectos, afrontó dificultades para la pronta atención, siendo nuestro objetivo contribuir a evitarlas mediante una planificación adecuada y a maximizar la eficiencia y eficacia del apoyo a la población afectada.

El Problema se refiere a la falta de centros seguros de atención y refugio de localización permanente y adecuadamente ubicados para que la población acuda a ellos en el menor tiempo posible en situaciones de desastres naturales en las diferentes localidades del país. En consecuencia no se dispone de personal y otros recursos asignados permanentemente para brindar atención en esos lugares de manera eficiente y eficaz.

Es objetivo del estudio proponer la ubicación de los refugios en lugares seguros y la cantidad óptima de recursos en cada refugio para la atención oportuna y en el menor tiempo posible, de los pobladores afectados.

La hipótesis de trabajo parte de la suposición que el conjunto de acciones dadas mediante modelos de localización, redes de Petri y simulación de redes de Petri, permitirán optimizar la atención de los afectados por un desastre natural en el sentido de ofrecer un lugar seguro de atención o refugio apropiadamente ubicado para acceder a este en el menor tiempo posible, con los recursos suficientes para atender a los afectados apropiadamente y bajo parámetros que garanticen su pronta atención.

Es de este modo que planteamos que la población afectada debe acudir a lugares seguros de refugio y atención de necesidades básicas (alimentación, vivienda, vestimenta, abrigo, aseo, atención médica), establecidos por la sede de defensa civil de cada lugar, cuyo conocimiento debe ser de permanente difusión entre la población.

Se trata de determinar la localización de los lugares seguros que son algunos nodos de la red conformada por el total de nodos que representan las diferentes áreas en las que reside la población. La localización final de estos lugares seguros y asignación de la población de cada área a estos lugares se determina mediante métodos exactos o mediante métodos heurísticos de localización. 
El problema de localización se enfoca como un problema de p-medianas y el criterio o medida de utilidad indirecta será en este caso el tiempo de inicio de la atención a la población que acude al lugar seguro, asociado a la menor distancia para acudir a ese lugar, medida que se desea minimizar. Luego de haberse ubicado los lugares de atención, se enfoca el problema de la atención eficiente y oportuna a los pobladores que la requieren, para lo cual se construye una red de Petri para cada centro de atención. En esta red se representa el proceso integral de atención y todas sus alternativas: registro de personas, atención médica, refugio, entre otros servicios.

La red de Petri resultante se simula para determinar las longitudes máxima y promedio de colas, tiempo promedio de espera, tiempo promedio en el proceso por persona y se realizan cambios en los parámetros para determinar su valor más adecuado para brindar la mejor atención posible. También se realizan cambios simulados en parámetros tales como tasa promedio de atención o incremento de puestos de atención en cada refugio y se determina el número óptimo de puestos de atención de cada tipo para minimizar el tiempo de atención a la población afectada que acude a un refugio determinado.

\section{Metodología}

Mediante el análisis de fuentes secundarias, especialmente genėradas por Defensa Civil y organizaciones no gubernamentales y publicaciones periodísticas, se estudian los datos referentes al desastre natural ocurrido, que en este caso particular son los datos referidos al sismo del año 2007 ocurrido en el Perú. Estas fuentes aún tienen datos en proceso o incompletos.

Como métodos de Investigación de Operaciones se aplican los Modelos de Localización para la ubicación adecuada de los refugios, Redes de Petri para la determinación del número óptimo de puestos de atención en cada refugio con la finalidad de brindar atención a la población afectada en minimizando el tiempo promedio de atención. Finalmente, usando la Simulación se procede a simular la red de Petri para observar el funcionamiento del sistema de atención y se modifica el sistema sobre el modelo hasta hallar la configuración más adecuada de servidores o puestos de atención en un refugio. Por cada refugio debe establecerse una red de Petri específica así como debe desarrollarse una simulación propia.

\subsection{Localización de lugares de atención}

Los problemas de localización han sido ampliamente tratados por diversos autores, en particular [5] y [7], utilizan el enfoque de las p-medianas que aplicamos en este estudio.

Respecto al problema de localización para este caso, se trata de un problema de las p-medianas capacitado, el cual se representa como un modelo de programación lineal entera binaria.

Minimizar $\sum_{i=1}^{n} \sum_{j=1}^{n} w_{i} d_{i j} x_{i j}$

Sujeto a:
(1) $\sum_{j=1}^{n} x_{i j}=1, i \in N$;
(2) $\sum_{j=1}^{n} x_{i j}=p, i \in N$;
(3) $\sum_{j=1}^{n} w_{i} x_{i j} \leq Q_{j} x_{i j}, i, j \in N$;
(4) $x_{i j} \in\left\{x_{i j}\right\} ; i, j \in N$;

Cada nodo $i$ representa un área de la ciudad donde se encuentra la población afectada y cada nodo $j$ representa un lugar seguro de atención o refugio, esto es, una mediana.

Las restricciones (1) y (2) garantizan que cada nodo que representa un área de la población afectada sea asignado a sólo una mediana o lugar seguro de atención y refugio. También garantizan que se 
definan p lugares de atención y refugio. Las restricciones (3) garantizan que se respete la capacidad de atención de cada lugar seguro de atención y refugio.

$\left[x_{i j}\right]=$ matriz de localización en la que:

$x_{i j}=1$ si la población ubicada en el área $i$ es asignada al lugar de atención y refugio $j ; x_{i j}=0$ si no lo es.

Asimismo, $x_{j j}=1$ si el nodo $j$ es una mediana o lugar seguro de atención y refugio; $x_{j j}=0$ si no lo es.

$N=$ conjunto de nodos que representa las áreas en que se ha dividido la localidad afectada; $N=\{1,2, \ldots, n\}$.

$w_{i}=$ población correspondiente al área i de la ciudad.

$Q_{j}$ representa la cantidad de población que puede ser atendida en el refugio o mediana $j$.

La solución a este problema se enfoca mediante el algoritmo de sustitución de nodos y alternativamente mediante algoritmos golosos.

\section{2. $\quad$ Redes de Petri}

Las redes de Petri permiten estudiar el problema como un sistema de eventos discretos a lo largo de un proceso. Construida la red de Petri se aplica la simulación para estimar el valor de un conjunto de variables endógenas que permiten evaluar el rendimiento del sistema y cómo mejorarlo a través de cambios en ciertos parámetros tales como el número de puestos de servicio que presten atención en cada lugar seguro de refugio y atención. En [1] y [8] se tratan la simulación de eventos discretos y las redes de Petri, respectivamente.

Las redes de Petri, son grafos que representan modelos de eventos discretos que ocurren a lo largo de un proceso en el que se desarrollan actividades. Las actividades se desarrollan en lugares y los eventos producen cambios o transiciones en las variables de estado del sistema de interés.

Consideramos de utilidad para este estudio el uso de las redes de Petri debido a que permiten representar características como el paralelismo y la sincronización, analizar cuantitativamente el sistema mediante el estudio de su estructura, representar diferentes niveles de abstracción, determinar todos los eventos que pueden desencadenarse al ocurrir un evento dado, entre otras facilidades.

Una red de Petri queda definida por, [8]: $R P=\left(P, T, A, W, M_{0}\right)$, donde:

$P=\left\{P_{1}, P_{2}, P_{3}, \ldots, P_{n p}\right\}$, es el conjunto de nodos lugar, que en el problema tratado son: colas para espera de atención, puestos en los que se atenderá a la población afectada que acudirá al refugio $j$; stocks o inventarios de medicinas, alimentos u otros medios de ayuda a la población afectada.

$T=\left\{T_{1}, T_{2}, T_{3}, \ldots, T_{n p}\right\}$ es el conjunto de nodos transición que representan eventos, tales como arribos de personas afectadas a los refugios, fin de la atención de otras personas, arribo de medios de apoyo.

$A=\left\{A_{1}, A_{2}, A_{3}, \ldots, A_{n p}\right\}$, es el conjunto de arcos de la red de Petri, tal que $A \subset(P \times T) \cup(T \times P)$, que representa el conjunto de vías a través de las que puede transitar la población.

$W=\left\{W_{1}, W_{2}, W_{3}, \ldots, W_{n p}\right\}=A_{i} \rightarrow\{1,2,3, \ldots, m\}, \forall A_{i}$ es la ponderación asociada a cada arco, en este caso representa el número de personas afectadas que pueden atenderse simultáneamente en el lugar seguro de atención o refugio.

$M_{0}=\left\{M_{1}, M_{2}, M_{3}, \ldots, M_{n p}\right\}=P_{i} \rightarrow\{1,2,3, \ldots, m\}, \forall P_{i}$ es el número de marcas o tokens iniciales en cada nodo lugar, en este caso puede representar el número de personas afectadas que se encuentran en una cola al inicio del proceso o el estado de una condición en un nodo lugar como, por ejemplo, si un puesto de atención está libre.

$P=\left\{P_{1}, P_{2}, P_{3}, \ldots, P_{n p}\right\}$ 
Haciendo uso de estas propiedades se construye la red de Petri para un lugar de atención y refugio, en la cual se representa el proceso integral de atención y todas sus alternativas: registro de personas, atención médica, refugio, entre otros servicios.

La red resultante se simula mediante simulación de eventos discretos, para determinar las longitudes máxima y promedio de colas, tiempo promedio de espera, tiempo promedio en el proceso por persona. Realizando cambios en los parámetros tales como tasa promedio de atención o incremento de puestos de atención en cada lugar de atención o refugio, se determina el número óptimo de estos puestos para minimizar el tiempo de atención a la población afectada que acude a un refugio determinado.

$\mathrm{Al}$ tratarse de un trabajo en proceso se ha procedido, para realizar el estudio, a generar escenarios para determinar la localización óptima de los refugios en una localidad hipotética, se ha supuesto que los pobladores afectados llegan a los puestos de atención de acuerdo a diferentes distribuciones de probabilidad, se ha dado un tamaño supuesto de población que acude a un refugio determinado y se ha probado la eficiencia y oportunidad de atención en el refugio. Los cambios en los parámetros han permitido verificar la capacidad de respuesta del centro hipotético de atención.

Un problema hallado bajo este estudio es que se debe disponer de personal capacitado en los diferentes servicios de atención que permanezca en el refugio o que pertenezca a instituciones de servicios tales como hospitales, centros asistenciales, compañías de bomberos asignados como servidores de estos centros en casos de emergencias.

\section{Discusión}

Los modelos de localización son medios muy útiles para la ubicación apropiada de centros de atención o refugio, de acuerdo a cada ciudad o centro poblado, existen diferentes enfoques que podríamos usar para determinar la ubicación más adecuada de los refugios en una ciudad, particularmente, consideramos que el enfoque de las p-medianas es apropiado para el problema que estamos tratando debido a que todos los nodos de la red no tienen los mismos atributos, existiendo dos tipos, aquellos en los cuales habita la población y aquellos en los que también habita una parte de la población pero que por sus características de seguridad pueden albergar un refugio.

Las redes de Petri son modelos que permiten representar situaciones que se desarrollan simultáneamente, permiten representar la sincronía entre eventos, los eventos que se desencadenan al ocurrir un evento determinado, las transiciones de estados, facilidad que no es factible representar con los modelos asociados a la teoría de colas o a la simulación tradicionalmente utilizada.

La Simulación asociada a las redes de Petri nos permite acercarnos mas al funcionamiento de los sistemas reales en los que ocurren eventos simultáneos como la llegada de varios pobladores afectados en el mismo instante, un evento como la llegada de un poblador afectado produce la ocurrencia de varios eventos tales como la ocupación del servicio de un médico, el inicio de la atención del poblador afectado, el uso de un equipo de cardiología, la ocupación de una camilla, la desocupación de una ambulancia, entre otros.

\section{Conclusiones}

4.1 Se busca optimizar la atención a las poblaciones afectadas en los casos eventuales que ocurran desastres naturales de diverso origen.

4.2 En estos casos la minimización de los tiempos de desplazamiento de las personas afectadas a los lugares de atención y refugio y de los tiempos de atención en esos lugares son los criterios 
relevantes que se toman en consideración para evaluar la efectividad y oportunidad de los servicios que deben brindarse.

4.3 Bajo las condiciones supuestas para fines de estudio se determinó que es posible brindar un servicio adecuado siempre que se encuentre con personal suficiente y capacitado, asignado a los refugios para atención de emergencias.

4.4 La presente es una propuesta cuyo impacto se verificará al aplicarse sobre los datos obtenidos sobre el sismo del año 2007, que aún se encuentran incompletos en las entidades pertinentes.

\section{REFERENCIAS BIBLIOGRÁFICAS}

[1] BANKS, J., CARSON, J.; NELSON B.L.. Discrete-Event Simulation. Prentice-Hall.1996.

[2] GUASCH M; CASANOVAS J.;Figueras J.. Modelado y Simulación. Ediciones UPC. Cataluña. 2002.

[3] KUROIWA, Julio. Ciencia y Tecnología. Memoria del Seminario Taller Internacional sobre Prevención y Atención de Desastres. Lima 2004.

[4] KUROIWA, Julio. Protección de Lima Metropolitana ANTE Sismos Destructivos. UNI y SE Comité de Defensa Civil. 1975.

[5] PIZZOLATO, Nélio. Localización de Escuelas Públicas. Pesquisa Operacional. Vol. 24. No 1, 2004.pp 111-131.

[7] SANTANA G. Participación comunal en la respuesta inmediata y rehabilitación para casos de eventos generadores de desastres. ONU; UNESCO; OEA; U.S. Oficina de Asistencia para Catástrofes. Caracas 2000.

[8] TEITZ, M Y BART,P. Heuristic Methods for Estimated Generalized Vertex Median of a Weighted Graph; Operations Research, vol 16, 1968, pp. 955-961.

[9] VERNON, M. .ZAHOEJAN J. LAZOWSKA E. A Comparison of Performance Petri Nets and Queueing Network Models. Proceedings on the International Workshop on Modeling Techniques and Performance Evaluation, Paris, 1987. 